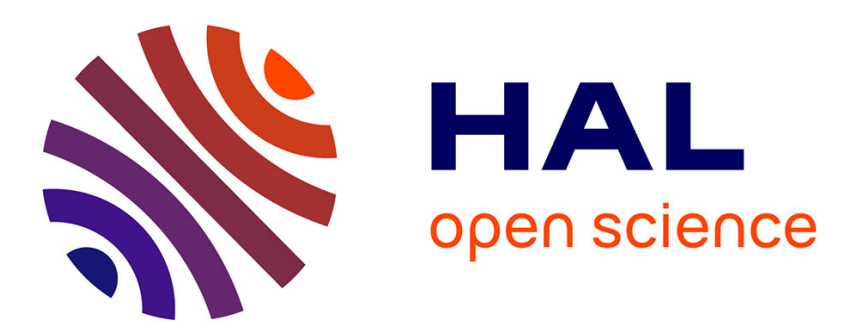

\title{
Analysis of the flow pattern modifications in a bubbly Couette-Taylor flow
}

A. Mehel, Céline Gabillet, Henda Djeridi

\section{To cite this version:}

A. Mehel, Céline Gabillet, Henda Djeridi. Analysis of the flow pattern modifications in a bubbly Couette-Taylor flow. Physics of Fluids, 2007, 19, pp.118101-1: 118101-4. 10.1063/1.2786584 . hal01208342

\section{HAL Id: hal-01208342 \\ https://hal.science/hal-01208342}

Submitted on 15 Feb 2017

HAL is a multi-disciplinary open access archive for the deposit and dissemination of scientific research documents, whether they are published or not. The documents may come from teaching and research institutions in France or abroad, or from public or private research centers.
L'archive ouverte pluridisciplinaire HAL, est destinée au dépôt et à la diffusion de documents scientifiques de niveau recherche, publiés ou non, émanant des établissements d'enseignement et de recherche français ou étrangers, des laboratoires publics ou privés. 


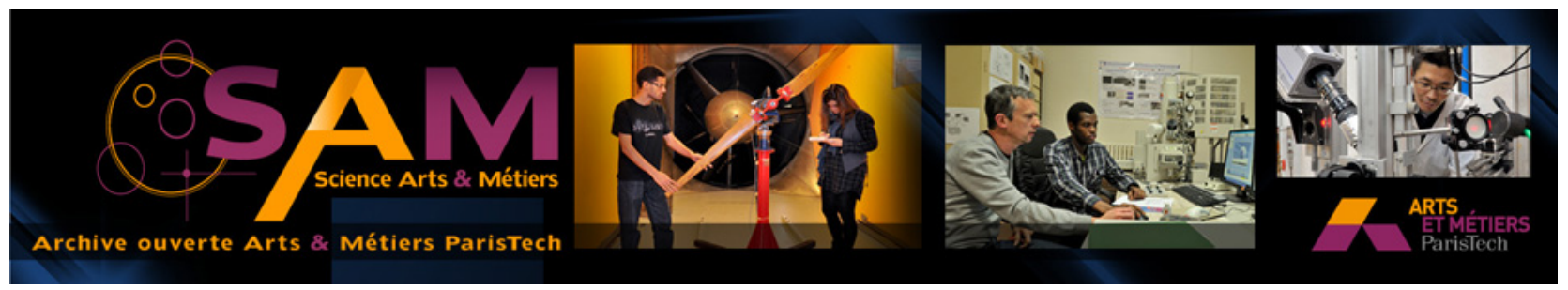

Science Arts \& Métiers (SAM)

is an open access repository that collects the work of Arts et Métiers ParisTech researchers and makes it freely available over the web where possible.

This is an author-deposited version published in: http://sam.ensam.eu

Handle ID: .http://hdl.handle.net/10985/10301

\section{To cite this version :}

Amine MEHEL, Céline GABILLET, Henda DJERIDI - Analysis of the flow pattern modifications in a bubbly Couette-Taylor flow - Physics of Fluids - Vol. 19, p.118101-1: 118101-4 - 2007 


\title{
Analysis of the flow pattern modifications in a bubbly Couette-Taylor flow
}

\author{
A. Mehel, C. Gabillet, ${ }^{\text {al }}$ and H. Dieridi \\ IRENav, Inrtitut de Recherche de I'Ecole Navale, Lamvéoc Poulmic, BP 600, 29240 Brest Arméex, France
}

\begin{abstract}
The aim of this Brief Communication is to discuss the bubble effect on the Couette-Taylor flow patterns in the transition from laminar to turbulent flow, especially in the weakly turbulent regime. It is shown that bubble location and local void fractions both in the vortices cores and in the near wall regions directly inffuence the axial wavelength. Bubbles trapped in the vortices tend to increase the vorticity and reduce the axial diffusivity. Bubbles near the wall contribute to "shear induced" turbulence depending on the void fraction gradient near the wall and the bubble size.
\end{abstract}

Studying flows between two coaxial circular cylinders is of much interest for chemical, biomedical, and nuclear applications. In this particular flow, increasing the rotution speed of the inner cylinder leads to the development of well known instabilities and turbulence takes place progressively. As far as the single-phase flow is concerned, the state of the art is very copious but very few studies deal with bubbly flow in this configuration.

Experimental studies have already been conducted in turbulent Couette-Taylor bubbly flows with superimposed axial flux. ${ }^{1,2}$ The arrangement of the bubbly phase depends on the ratio between the Taylor number $\mathrm{Ta}$, characteristic of the rotation rate, and the Reynolds number, characteristic of the injection axial fiux. Nevertheless, in this situation, the bubble effect on the flow patterns cannot be decorrelated from the axial flow effect that tends to reduce the size of the Taylor vortices and thus the axial wavelength.

In order to investigate the interactions between bubbles and the flow structures, it is necessary to conduct studies without superimposed axial flow in the transition from laminar to turbulent flow. The question we try to answer is: What is the bubbles' location and the void fraction effect on the Taylor vortices intensity, the wall shear stress, and the tmasition to higher order instabilities? To this end, measurement were conducted in two complementary experimental devices detailed in Refs, 3-5.

Detniled results obtained for the wavy vortex flow and modulated wavy vortex flow regimes $(188<\mathrm{Ta}<543)$ are discussed in depth in Ref. 3 . It is put into evidence that millimetric vapor bubbles generated by pressure drop (cavi tation) are periodically ammged. They are located either in the Taylor vortices for $\mathrm{Ta}<300$ or in the outflow regions for larger Taylor number. In this latter situation, there is an increase in the axial wavelength and a premature transition to the third instability induced by the migration of the bubbles in the outflow regions.

However, in order to investigate the correlation between the flow pattern modifications and the local dispersed phase

"Electronic mail: gahillet Qecole-navale.f

Present address: Laboratoire de Physique des Octans, 6 Avenue Le Gorgen, B.P. B09, 29285 Tinest Cedex, France. characteristics (void fraction, bubble size, and drift velocity), a larger experiment was built that makes it possible to introduce a dual fiber-optic probe and to perform measurements for higher instabilities. In Ref. 4, void fraction and velocity profiles measured in this new experimental device are presented and discussed according to the bubble size: millimetric bubbles (cavitation) or submillimetric bubbles (ventilation). In Ref. 5, further measurements obtained for millimetric bubbles with another ventilation procedure ane displayed. The changes in the flow patterns are obviously more pronounced when the bubble size is increased. The objective of the present Brief Communication is to sammarize and bring together results obtained in weakly turbulent flow for millimetric bubbles by the way of different procedures. ${ }^{45}$ It gives an extended analysis of modifications in axial wavelength, vorticity, wall shear stress, and diffusivity, as a function of local phase characteristics, through a dimensional approach.

The geometry of the experimental device is characterized by the following nondimensional parameters (gap: $d / R_{i}=0,1$; height: $\Gamma=L / d=40$ ) with $d=R_{n}-R_{i}=20 \mathrm{~mm}$. The Taylor number is defined as $\mathrm{Ta}=\sqrt{\Omega_{i}^{2} R d^{3} f v^{2}}$, where $\Omega_{i}$ is the inner cylinder angular velocity, $R_{i}$ the radius, and $\nu$ the viscosity of the experimental fluid, which is a mixture of water and glycerol. Figure 1 shows a general schematics of the apparatus. The characteristics of the single-phase flow patterns are given in Ref. 4 . In this study, special attention was paid to the quasiperiodic regime at $\mathrm{Ta}=780$, and the weakly turbulent regime at $\mathrm{Ta}=1000$. From now on, subscript $40 "$ is used to denominate the single-phase flow patterns.

The bubbles were generated either by air injection at the bottom of the apparatus associated with a pressure drop (gaseous cavitation) or by ventilation at the unper free surface. The bubble size depends on the generation procedure: millimetric bubbles for cavitating flows, both millimetric and submillimetric bubbles for ventilated flows (denoted "CF" and "VF", respectively). The ventilation procedure is slightly different than the one used in Ref. 4, where only submillimetric bubbles were trapped in the gap.

For CF and VF, millimetric bubbles are localized both in the outflow regions near the inner cylinder and in one in two 


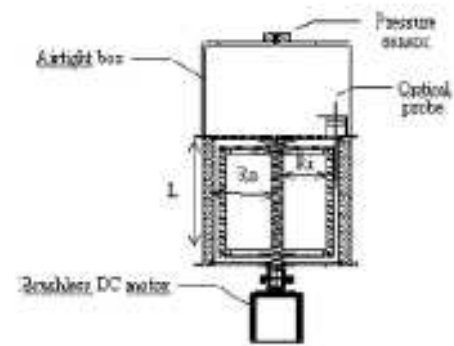

HG. 1. General schematies of the apparatus.

Taylor vortex cores, as can be seen in Fig 2 . In the vortex cores, millimetric bubbles of size $d_{n} / d=0.16-0.18$ are spherical shaped. In the cutflow region, millimetric bubbles are elongated in the direction of the azimuthal flow (large axis of the ellinsond $l_{\operatorname{an}} / d=0.3$ ). For the ventilated flow, the obtained submillimetric bubbles $\left(d_{n} / d=0.04\right)$ are localized in the outflow regions. In Table I, contribution of millimetric and submillimetric bubbles to the void fraction are distinguished by a special treatment of the optical probe signal. ${ }^{5}$ it is necessary to discriminate the respective contributions in order to analyze specific effect of millimetric bubbles on the flow patterns. The axial wavelength can be linearly stratified (SF) or homogeneous (HF), according to the bubble generation procedure. For cavitation, the flow is stratified during the pressure decrease and reorganizes into a homogeneous flow as soon as pressure is stabilized. For ventilation, the flow remnins stratified, Local measurements were performed in the third bubble string below the free surface. The void

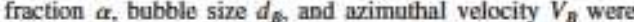
determined with a dual fiber-optic probe. The liquid azimuthal and axial velocity components $(w, w)$ were characterized by laser Doppler velocimetry.

Figure 3 shows that the relative variation in the axial wavelength evolves linearly with the difference between the void fraction in the vortices core and the void fraction in the outflow region near the inner cylinder. As was suggested in Ref. 4 , it is now obvious that bubbles located preferentially in the vortices' core tend to reduce the vortex size, whereas bubbles located preferentially in the outflow region tend to increase this size.

Let us now suggest the physical understanding. To achieve this, it is necessary to have a look at the bubbles'
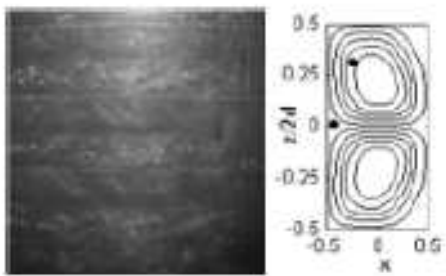

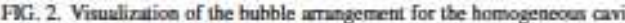
tuting flow [ $x$ denotes the normalized rodial position $x=r / d-(\mathrm{Ri} / d+10.5)]$.
TABIE 1. Bubble cuntribution to the voud fraction and wall shear stress (void fraction gratient and buhble drift velocily are determined at $x=-0.375$ in the thired bubble string below the froe surface, $n_{s}$ is the bubble string number in the gap).

\begin{tabular}{|c|c|c|c|c|c|c|}
\hline & $\begin{array}{c}\mathrm{Ta} \\
\mathrm{a} i \text { (nos) }\end{array}$ & $\begin{array}{l}\text { Flow } \\
\text { putiern }\end{array}$ & $\begin{array}{l}\alpha(\%) \\
\text { care }\end{array}$ & $a_{\text {imhis }}\left(\xi_{0}\right)$ & $\frac{V_{0}-V_{g}}{V_{a}}$ & $\frac{r_{w}-r_{w n}}{\rho \nu V_{g} / d}$ \\
\hline \multirow{6}{*}{$\mathrm{CF}$} & \multirow{3}{*}{$\begin{array}{l}780 \\
1.56\end{array}$} & $n_{s}=15$ & & & & \\
\hline & & SI & 0.35 & 0.077 & $0.2 \mathrm{x}$ & 0.65 \\
\hline & & HF & 0.12 & 0.045 & 0.28 & 0.38 \\
\hline & \multirow{3}{*}{$\begin{array}{l}1000 \\
1.76\end{array}$} & $n_{s}=16$ & & & & \\
\hline & & SF & 0.75 & 0.139 & 0.26 & 0.81 \\
\hline & & HF & 0.3 & 0.07 & 0.21 & 0.41 \\
\hline \multirow{4}{*}{ VF } & & & & millimetric: & & \\
\hline & $\begin{array}{l}780 \\
3.07\end{array}$ & $\begin{array}{c}n_{s}=14 \\
\mathrm{SF}\end{array}$ & 0.21 & $\begin{array}{c}0.062 \\
\text { suhmillimesnic } \\
0.21\end{array}$ & 0.24 & 0.33 \\
\hline & & & & millimetric: & & \\
\hline & $\begin{array}{l}1000 \\
3.07\end{array}$ & $\begin{array}{c}n_{x}=14 \\
\text { SF }\end{array}$ & 0.23 & $\begin{array}{c}0.079 \\
\text { suhmillimetric } \\
0.36\end{array}$ & 0.19 & 0.27 \\
\hline
\end{tabular}

effect on the vorticity when bubbles are trapped in the vortices. Values of the local pressure Laplacian can be used to distinguish "vorticity dominated" regions (positive values) from "strain dominated" regions (negative values). To calculate the pressure Laplacian, an analytical solution for the velocity field must be known. For this purpose, a fourthorder Fourier decomposition (Davey's expansion theory extended to larger Taylor numbers ${ }^{6}$ ), applied to axial profiles of measured values of axial and azimuthal mean velocity components, was achieved. Davey's coefficients were then radially fitted by polynomial curves. The mean radial velocity $u$ was deduced from the continuous equation. Errurs induced by this decomposition are deduced from comparison between experimental and analytical data. Relative errors are less than $5 \%$ and $2 \%$ for axial and arimuthal velocities, respectively.

Figure 4 displays the isocontours of pressure Laplacian for single and cavitating flows at $\mathrm{Ta}=780$. With the presence of the bubbles, the global vorticity increases and the location of the global maximum shifts towards the inner cylinder and farther from the outflow region. This is in agreement with the capture of the bubbles at the position $(z / \lambda=0.34, x=r / d$ $-\left(R_{i} / d+0.5\right)=-0.22$, as can be seen in Fig. 2) which tends to augment the local vorticity, with a reinforcement in the inflow and a raduction in the outflow of the shear between the vortices. The buoyancy leads to an upward shift of the vortex core. Let us consider the final location of the bubbles in the
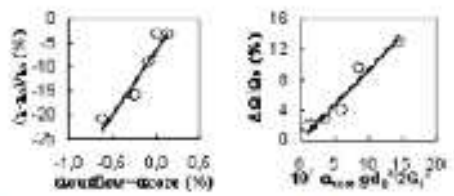

FGG, 3. Relative variation in atid wavelength and vorticity indwed by the huhbles a a finction of vnid fnartion due to millimetric buhbies and nor. malized monentum exchunge, respectively. 


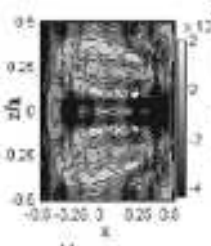

(i)

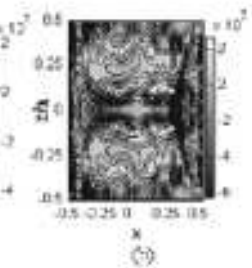

$\Leftrightarrow$
FXC. 4. Pressure 1 splacian obtained by modelization of the velocity field ut T2-780: (a) single-phuse flow and (b) hamogenecus cavitaling bow.

vortex $r_{a x}$, with $G$ and $R$ the vortex strength and radius. A dimensionless analysis shows that $r_{\mathrm{eq}} / R$ depends on three parameters such as $\left(d_{D} / R, d_{B}{ }^{3} g / G^{2}, R_{e_{D}}=G d_{B} / \nu R\right)$. The second parameter is the ratio between buoyancy and inertia of fects, $\mathrm{Re}_{g}$ is the ratio between inertia and viscous effects. In the present study $d_{n} / R$ and $R_{n}$ are quite constant (equal to $0.21 \pm 5 \%$ and $52 \pm 15 \%$, respectively). Assuming that the bubble drift velocity at the equilibrium position can be deduced from the vortex strength, the ratio $6 C_{d} r_{\mathrm{eq}} / 8 d_{n}$ representative of the ratio between drag force and inertia forces (lift, tchen, and added mass) applied to the bubbles is of order $1 . C_{d}$ is the drag coefficient deduced from Moore's correlation. ${ }^{7}$ When we consider the balance between the forces including buoyancy, it can be shown that $r_{\mathrm{eq}} / R$ evolves as $g d_{n}^{3} / G^{2}$, in agreement with a shift of the final location outward according to the ratio between buoyancy and inertia. $r_{e q}$ obviously augments when bubble diameter increases or vortex strength decreases. The force by unit volume induced by the bubbles trapped in the vortex $\Delta F_{i}$ is the consequence of added mass, lift, and drag forces and evolves as $\alpha_{\text {oeed }} \rho G^{2} r_{\mathrm{eq}} / 4 \pi^{2} R^{4}$. Hence, the bubbles lead to an increase in the pressure gradient that can be normalized by the global initial pressure gradient. When considering the formulation of $r_{\mathrm{eq}} / R$, it yields

$$
2 \frac{\Delta \Omega}{\Omega_{0}}=\frac{\Delta(\operatorname{grad} P)}{(\operatorname{grad} P)_{0}}=\frac{\Delta F_{b}}{(\operatorname{grad} P)_{0}}-\alpha_{\operatorname{cur}} \frac{g d_{n}{ }^{3}}{G^{2}} .
$$

The bubble induced relative vorticity is now deternined assuming that $\Delta \Omega / \Omega_{0}=\Delta v_{t} / v_{d 0}-\Delta \lambda / \lambda_{0}$, where $v_{t}$ is the tangential velocity deduced from the measurement of mean axial velocity profiles at the radial position $x=0.25$. The evoIution of bubble induced vorticity with respect to $\Delta F_{b} /$ (grad $\left.P\right)_{0}$ is plotted in Fig. 3. It is interesting to note that the vorticity expands almost linearly with the void frac tion in the core and the ratio between buoyancy and inertia. The bubble capture in the core is responsible for an increase in vorticity and pressure gradients in order to compensate for bubble buoyancy. This is in agreement with a reduction in the vortex size, depending on the bubble residence time and the bubble number in the vortex, as was expected by Gopalan and Katz ${ }^{\mathrm{K}}$

When submillimetric bubbles are localized in the outflow region near the inner cylinder, there is an increase in the axial wavelength, associated with a decrease in the vorticity of the vortices (ventilated flow at the bottom of the apparatus). Nevertheless, when millimetric bubbles are trapped in the outflow region, the increase in the vortex size can be balanced by the augmentation of vorticity due to bubble capture in the core of the vortices (top of the apparatus in cavitating and ventilated flows).

In the single-phase Couette-Tuylor flow, the increase in the axial wavelength is observed when turbulence develops and it is linked to the increase in the wall shear stress. ${ }^{9}$ This conjecture is worth being checked in two-phase flow by analyzing the velocity gradient of the liquid near the wall in the outflow region. In Refs. 4 and 5 , it is shown that in this region, bubbles move slower than the liquid in the arimuthal direction. This is due to their migration process, which mukes them move from the opposite side of the gap towards the inser rotating cylinder. Therefore, the arimuthal bubble drift velocity $\left(V_{N}-V\right)$ leads to a decrease in the mean azimuthal velocity of the liquid near the inner cylinder $\Delta V / V_{0}$.

Let us now consider a simple model that correlates $\left(V_{B}-V\right)$ and $\Delta V$. Based on a "two-fluid" approach, the change in the azimuthal momentum is due mainly to the bubble induced momentum associated with drag effects of millimetric bubbles. Under these considerations, the azimuthal liquid velocity is deduced from the following expression:

$$
V=V_{n}+\frac{3 k_{\theta}}{4 d_{n}^{2}} \frac{\nu r}{U_{0}} \alpha_{n}\left(V_{R}-V_{0}\right),
$$

where $\alpha_{n}$ is the contribution of millimetric bubbles to the void fraction, $k_{\theta}$ is the ratio between the drag coefficient and the bubble Reynolds number $\left(\left|V_{B}-V_{0}\right| d_{D} / \nu\right)$, and can be determined with Moore's correlation. ${ }^{7} V_{0}$ and $U_{0}$ are referred bere to the mean azimuthal and radial velocity components of the undisturbed flow (i.e., the single-phase flow). For the bubble Reynolds number at stake $(-85)$, had we taken into account for interface contamination, the drag coefficient ${ }^{10}$ would have been greater by a factor $C \sim 2$. Another issue is that we have to consider the contribution of the bubble indoced velocity gradient in the arimuthal momentum exchange:

$$
\frac{\Delta V}{V_{0}}+\frac{r}{V_{0}} \Delta\left(\frac{\partial V}{\partial r}\right)=C \frac{3 k_{\theta} \nu r}{4 d_{D}^{2} U_{0}} a\left(\frac{V_{B}-V_{0}}{V_{0}}\right) .
$$

Near the inner cylinder, at $x=-0.375$, experimental data ${ }^{4}$ show that

$$
\frac{r}{V_{0}} \Delta\left(\frac{\partial V}{\partial r}\right)=-0.7 \Delta\left(\frac{\Delta V}{V_{0}}\right) .
$$

With this approximation, the bubble induced azimuthal velocity can be expressed as

$$
\frac{\Delta V}{V_{0}}=\frac{1}{1-0.7} C \frac{3 k_{\theta}}{4 d_{B}{ }^{2}} \frac{\text { or }}{U_{0}} \alpha_{B} \frac{\left(V_{B}-V_{0}\right)}{V_{0}} .
$$

The validity of the "two-fluid" approach can be checked in Fig. 5. $\Delta V I V_{0}$ near the inner cylinder deduced from experimental data at $x=-0.375$ shows a quite linear correlation with respect to $\alpha_{n}\left(V_{n}-V_{0}\right) / V_{0}$, the slope being ten times as great as 

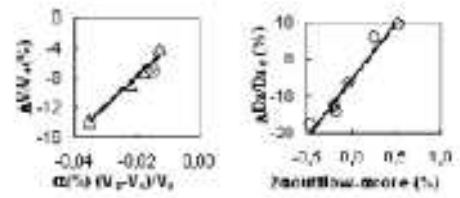

FG. 5. Relative variation in mean arimuthal velocity and axial diffusivity induced by the bubbles as a finction of void fraction and drift velocity, near the wall in the oufllow. $(0-\diamond)$ Ventilated flaws; $(\Delta-\Delta)$ cavitzing flows; $(0-0)$ all Blowi.

$$
\frac{3 k_{b}}{4 d_{R}^{2}} \frac{\nu r}{U_{0}} \alpha_{R} \frac{\left(V_{R}-V_{0}\right)}{V_{0}}
$$

As expected from this, the shear stress can be written as

$$
\begin{aligned}
\tau_{r \theta}(r)=\tau_{r \omega 0} & +10 \frac{3 k_{\theta}}{4 d_{D}^{2}} \frac{\nu r}{U_{0}} \mu \\
& \times\left[\alpha_{D} \frac{V_{B}-V_{0}}{r}-\alpha_{B} \frac{\partial\left(V_{D}-V_{0}\right)}{\partial r}-\frac{\partial \alpha_{B}}{\partial r}\left(V_{B}-V_{0}\right)\right] .
\end{aligned}
$$

The wall is expected to play a repellent role for the bubbles; "the void fraction at the inner cylinder wall is thas considered as negligible, whereas the radial gradient of the void fraction has a significant positive value. The expression of the wall shear stress yields

$$
\tau_{w}=\tau_{w 0}-10 \frac{3 k_{0}}{4 d_{B}^{2}} \frac{\nu R_{i}}{U_{0}} \mu\left(V_{R}-V_{0}\right) \frac{\partial \alpha_{B}}{\partial r} r=R_{i}
$$

Taking into account that the bubble velocity is less importan then the liquid velocity near the inner wall, the wall shear stress appears to be greater than the one obtained in singlephase flow. Thus, a higher value of the void fraction near the inner cylinder is expected to lead to an increase in the shear stress. The "bubble induced" wall shear stress determined in the outflow from Eq. (3) is reported in Table L. Note that it is underestimated in ventilated flow becuuse the contribution of submillimetric bubbles is expected to play a role in the very near wall region.

The bubble induced wall shear stress contributes to increase the production of turbulence near the wall. For the axial velocity component, additional turbulent kinetic energy was observed in the entire gap. ${ }^{5}$ As it has been shown in single-phase flow, ${ }^{12}$ that the turbulent axial diffusivity $D_{\mathrm{Z}}$ expands linearly with the axial wavelength, the relative variation in diffusivity can thas be roughly estimated following

$$
\frac{\Delta D_{Z}}{D_{D 0}}=\frac{\Delta \lambda}{\lambda_{0}}+\frac{\Delta w_{\text {ms }}}{w_{\text {ms } 0}}
$$

$w_{m g}$ is the rms axial velocity component measured near the inner cylinder in the oulflow region. $\Delta D z / D z_{0}$ is displayed in
Fig. 5 as a function of both the global void fraction in the outflow and the void fraction in the core of the vortices. It can be seen that the diffusivity is proportional to $\left(2 \alpha_{\text {outhow }}\right.$ $-\alpha_{\text {cuel }}$, in agreement with a quasilinear growth of $w_{\mathrm{ms}}$ with $\alpha_{\text {ounse }}$ or $(\partial \alpha / \partial r)_{R_{l}}$ in the outflow and a linear dependence of $\lambda$ with ( $\alpha_{\text {nuthow }}-\alpha_{\text {care }}$ ). Hence, bubbles localized near the wall in the vutflow region are supposed to increase the axial diffusivity, whereas bubbles captured inside the Tayior vortices are expected to reduce this quantity.

To conclude this Brief Communication, for the bubbly Taylor-Couette flow in the quasiperiodic and in the weakly turbulent regimes, it is demonstrated that modifications in the flow patterns depend on the bubble location in the gap, according to their size and that they are correlated with the local void fraction. For bubbles located near the wull, drift velocity is responsible for the homogenization of aximuthal momentum in the gap and the increase in the wall shear stress. This is in agreement with results expected in flows where bubbles injected outside the boundary layer bring closer to the wall and exhibit a void fraction peak. As a conclusion, bubbles localized in the outflow region contribute to increase the axial wavelength and to develop turbulence by shear induced turbulence. On the contrary, bubbles trapped in the core of the Taylor vortices are expected to stahilize the flow and increase the vorticity.

'Y. Shiomi, H. Kutsuma, K. Nkagawa, and M. Otywa, "Two-phase fllow in an unnulus with a roating imner cylinder (flow puttern in bobbly flow negion)," Nucl. Eng. Des. 141, 27 (1993)

${ }^{2} \mathrm{~K}$. Mikhen, I. Funtaine, und I. E. Wesfreid, "Highly turbulent CouetteTuylor bebbly flow potiems," I. Fluid Mech, 422, 55 (2000).

${ }^{3}$ H. Djeridi, C. Gabillet, and I. Y. Billurd, "Two-phese Couetle-Taylor Bow: A mogement of the dispersed phave and effects on the flow structernes" Phys. Fluids 16, 12k (2004).

${ }^{4} \wedge$. Mehel C. Gabilles, and H. Djeridi, "llubble effect on the structures of weakly turbelent Coucte-Tuylor Bow," 1. Fuits Eng. 128, 819 (2006)

"A. Mebel "Fiude experimentale d"un ecuulement diphrsique de TiylorCouetie," P.D. thesis, Foole Centrale de Nanks and Univenite de Nantes, 2006.

"A. Devey, R. C. Di Prime, and J. T. Stuart, 40 n the stahility of Taylor vortices," I. Fuid Mech. 31, 17 (1968).

7D. W. Moure, "The boundary layer on a spberical gas butble," 1. Flind Mech. 16, 161 (1963).

"S. Gopulan and J. Kuth, "Effect of entrined bubbles on the structure of varter rings," J. Fluid Mech, 397, 171 (1999).

"G. Cognet, "Les elapes vers hil urbulence dams recoukment de Taylor. Couelie entre cylindres couriaux," I. Mec. Theor. Appl., special issos, p. J (1954)

${ }^{10}$ T. Marworthy, C. Ginann, M. Kurien, and F. Durst, "Firperiments on the rise of uit bubbles in clean viscous liquid" I. Fluid Mech. 321, 421 (1996).

"D. Legendre and J. Magnandet, "The fift force on a spherical hubtile in a viscoes linear sheur flow," I. Fleid Moch. 368, 81 (1998).

${ }^{17}$ W. Y. Tam and H. L. Swinney, Mass tratspoet in tuthulent CouetieTaylor flow," Phys. Rev. A 36. 1374 (1987). 